\title{
Slotting Optimization Algorithm of the Stereo Warehouse
}

\author{
JunHui Wu, TongDi Qin \\ Institute of Modern Agricultural Science \& Engineering \\ Tongji University \\ 4800 Caoan Hwy, Jiading, Shanghai, P.R. China \\ E-mail: junhui_wu@163.com \\ qintongdi@yahoo.cn \\ Qiang Zhou \\ Shanghai Urban Green Engineering Co., Ltd \\ 1688 Jianchuan Hwy, Shanghai, P.R. China \\ E-mail: zhou.qiang@dushigreen.com \\ corresponding author
}

\begin{abstract}
In order to solve the problem of slotting optimization management with automated storage and retrieval system (AS / RS), the genetic algorithms of the slotting optimization process with stereo warehouse were discussed. The process of the slotting optimization was designed by the real-number coding. The mathematical models of the slotting optimization and cargo shipping time were built, and the simulation calculation of the models was done. The stability of the shelf and the efficiency of the accessing goods were significantly improved by the optimization simulation of the distribution of cargo space, after optimizing the storage order. The algorithms of the slotting optimization with stereo warehouse could effectively enhance the frequency of the stock in \& out, increasing the revenue.
\end{abstract}

Keywords-automated storage and retrieval system; automated warehouse; slotting optimization; genetic algorithm; mathematical models; simulation calculation

\section{INTRODUCTION}

With the development of manufacturing and warehousing logistics industry, Slotting optimization management closely related to the productivity, shipping accuracy, inventory turnover, storage time, order fulfillment the time and storage density is given increasing emphasis. Slotting optimization management with automated storage and retrieval system (AS / RS) has become one of the key indicators of the automated warehouse. Slotting optimization management pursues to achieve the optimal layout of cargo space, mastering the available goods changing, fast efficiency of the stock in \& out, so to maximize cost savings. Therefore, slotting optimization becomes the primary task of the AS / RS operations, and has an important place in the management of AS / RS.

\section{The OVERVIEW OF THE SLOTTING OptimizATION}

Slotting optimization is dynamically reconfiguring the cargo space of the goods in the warehouse based on the inventory capacity of the stereo warehouse and unexpected variability to improve the frequency of the stock in \& out of

\author{
Jie Chen, HuiPing Si, KaiYan Lin \\ Institute of Modern Agricultural Science \& Engineering \\ Tongji University \\ 4800 Caoan Hwy, Jiading, Shanghai, P.R. China \\ E-mail: chenjie18@yahoo.com.cn \\ sihuiping@tongji.edu.cn \\ ky.lin@163.com \\ ChiBin Zhang \\ Institute of Mechanical Engineering \\ Southeast University \\ 79 Suyuan Hwy, Jiangning, Nanjing, P.R. China \\ E-mail: chibinzhang@yahoo.com.cn
}

the automated storage and retrieval system, conveniently completing the operations of saving and shifting library, reducing the time of store or the selection operation, reducing the cost of warehouse operations, reducing the loss of goods in the process of storing and handling, and ultimately increasing revenue purposes[1][2].

\section{A. The Principles of Slotting Optimization}

The optimization principle of cargo space allocation for the automated warehouse: random storage, specified the storage, classified storage. Randomly pick commonly uses the rules of longest wait time limit, the nearest neighbor (NN), the shortest leg, the correlation of the product storage, balance workload, and convenience the stock in \& out[3][4]. The selected rule of inventory and picking up for the automated warehouse usually is in accordance with the firstcome-first-served (FCFS) rules.

\section{B. The Steps of Slotting Optimization}

Slotting optimization is related to the types of storage goods of the entire AS / RS and running time, some time to go through the running, and the basic operating data as well as goods data are got. System design is completed according to the algorithm steps [3].

The usual process of slotting optimization is shown in Fig.1:

Data analysis of AS / RS foundation running

Determine the objectives and constraints of the slotting optimization

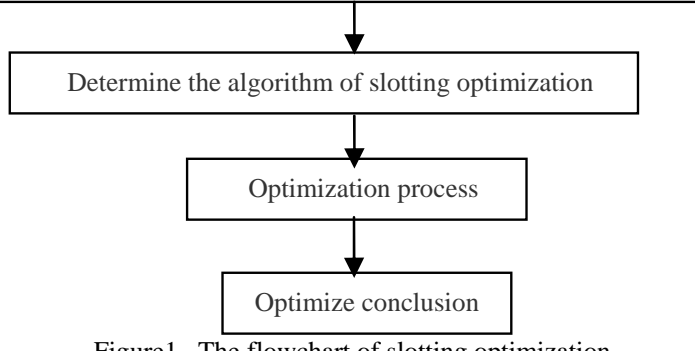

Figure1. The flowchart of slotting optimization 
(1) AS / RS data analysis mainly contains the analysis of cargo space characteristics, goods demanding information and product data file.

(2) Determine the objectives and constraints of optimization. The ultimate goal of slotting optimization is to reduce costs, which can be achieved by improving productivity and minimizing useless exercise. The effect of optimization can be assessed by operating efficiency of balancing the workload of operating unit, improving picking efficiency, reducing replenishment workload, shortening the picking distance. Therefore, slotting optimization is the problem of multi-objective decision.

(3) Determine the algorithm. Algorithm is a key part of the entire slotting optimization.

(4) Optimization process. The optimization process needs to establish a corresponding mathematical model, and uses appropriate language to express the algorithm, then debugs and tests. The accurate and effective cargo space optimization strategy is provided for the logistics center.

\section{The Algorithm OF SlOtTING Optimization}

More optimization methods and ideas expand from two aspects, one is the shortest path to study the picking goods of AS / RS, the other is to study the slotting optimization. The optimization algorithm is using genetic algorithm to solve the optimization problem of cargo space [5] [6] [7].

The genetic algorithm is a computational model of simulating the natural selection of Darwin's biological evolution and the biological evolution process of Gregor Mendel's genetics, and is the method of simulating natural evolutionary process with searching the optimal solution by [8][9].

The general process of the genetic algorithm[10][11]: (1) Determine the coding scheme of chromosome, coding and producing a set of initial population; (2) Calculate the fitness of each individual in the population, structuring the fitness function; (3) Select the individual in accordance with the genetic probability corresponding to fitness; (4) The cross operation; (5) The mutation operation; (6)Repeat(2)(5)Operation until meet the end conditions of algorithm.

The basic process of the algorithm, as shown in Fig. 2:

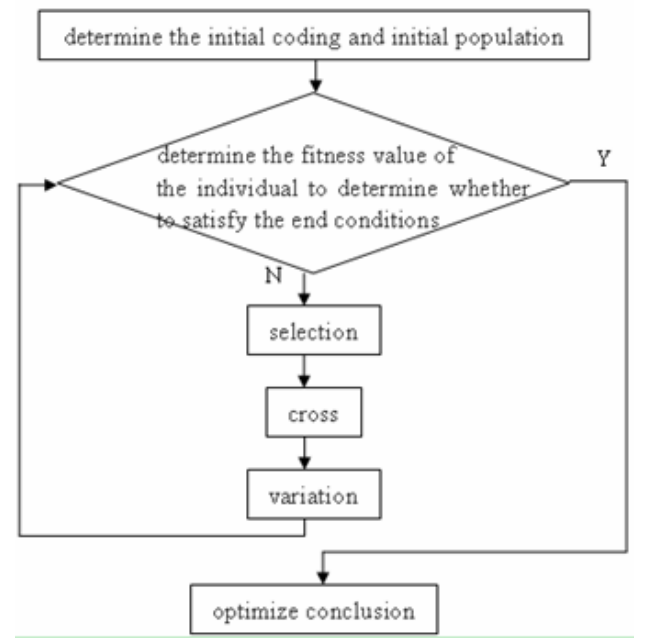

Figure2. The flowchart of genetic algorithms

\section{The Mathematical Model of Slotting OPTIMIZATION}

In this article, shelves are removable, but the cargo space is relatively fixed, so the optimization problem of cargo space is similar to the fixed cargo space shelves. Two optimization goals of shelf stability and accessing efficiency of cargoes are also involved. In the process of building mathematical model, it is assumed that the shelf system is in accordance with the principle of top-light to complete storage operations, and the stability of shelf obtains effective support. To support the goals of accessing efficiency, the storage port is the cargo space nearest from the stock in \& out station as was expressed earlier.

It is assumed that the total of automated warehouse is $p$ column $q$ layer, $i$ layer number, $j$ column number, then the cargo space of the $i$ layer $j$ column denoted as $(i, j),(i=1,2 \ldots$, $p ; j=1,2 \ldots, q)$, the shipping location is recorded as $(0,1)$. In the system of entire shelf, the cargo box weight of each cargo space is the same, and its length and width are consistent. Slotting optimization based on the strength and stability and the accessing efficiency of the shelf can be described as [12] [13]:

$$
\left\{\begin{array}{l}
\text { MinS }=\sum_{i=1}^{p} \sum_{j=1}^{q} m_{i j} \cdot n_{i j} \cdot j x_{i j} \\
\text { MinT }=\sum_{i=1}^{p} \sum_{j=1}^{q} t_{i j} \cdot x_{i j}
\end{array}\right.
$$

Where formula (1), $x_{i j}$ is 0 or $1, m_{i j}$ and $n_{i j}$ are the quality and quantity of the cargo space goods with $i$ column $j$ layer, in this paper, $n_{i j}$ is 1 . After ignoring the start time of the shelf level motor and the start and stop time of the vertical lifts, $t_{i j}$ is the time of the $i$ layer and $j$ column goods transporting to the shipping port, which is calculated as follows:

$$
t_{i j}=\frac{L \cdot i}{V_{x}}+\frac{H \cdot(j-1)}{V_{y}}
$$

Where formula (2), $V_{x}$ is the average speed of the shelf horizontal running, and $V_{y}$ is the average running speed of the vertical conveyor, and $L$ is the length of the cargo space, and $H$ is the height of the cargo space.

\section{Optimization Process of the SLOtTING}

A. code

Some literature studies suggest that the low hex encoding genetic algorithm is generally superior to the high hex encoding genetic algorithm in the robustness of the results of the search efficiency and optimization [14]. In this article, the optimization problem of the cargo space is the multiobjective and high precision continuous function optimization problems, and with a larger solver scale. However, coding method of the real number is more applicable to search within a larger space compared with the binary code, and a larger range of numbers can be represented effectively, significantly improving the operation 
efficiency of the genetic algorithm [8]. Therefore, in this paper, the real number to be encoded is used.

In the physical presence, cargo space is a threedimensional grid problem. The conventions of the convenient coding:

(1) Stereo shelves with $p \times q$ matrix to be encoded; (2) The cargo space with row $i$ and column $j$ of the matrix is $a_{\mathrm{ij}}$; also, each element in the matrix represents a cargo space, and encoding is a one-to-one encoding; (3) The integer 1, 2, $3 \ldots$ $\mathrm{n}$ represent as the corresponding location of the cargo in the cargo space matrix, and 0 in the cargo space is free; (4) A combination of cargo space corresponds to a chromosome, as follows:

$$
\begin{aligned}
& {\left[\begin{array}{ccccc}
1 & 2 & 3 & 4 & 5 \\
10 & 9 & 8 & 7 & 6 \\
11 & 12 & 13 & 14 & 15
\end{array}\right]} \\
& {\left[\begin{array}{ccccc}
1 & 2 & 3 & 0 & 0 \\
10 & 9 & 8 & 7 & 6 \\
11 & 12 & 13 & 14 & 15
\end{array}\right]}
\end{aligned}
$$

The matrix shown in formula (3), each cargo space is placed the cargo. Some cargo space in the following formula (4) is 0, which expresses as idle cargo space.

\section{B. The Initial Population}

According to the structure of the stereoscopic warehouse and operating data, the required number of the cargo space is obtained, there is part of empty cargo spaces to form the matrix, and then initialize the data in the matrix. Initialization data is randomly selected, which can ensure diversity of population data maximum, and is conducive to the generation of good genes.

Initialization population can randomly generate a certain number of individuals, and then pick out the best individuals added to the initialization groups and constant iteration, until the number of individuals in the initial population to meet the requirements. In the initial selection process, use the norm to measure the differences between the various chromosomes. This method is to determine a chromosome as a basis, to seek the distance between other chromosomes and reference chromosome in the population, so that it is greater than a determined value, which enables a larger difference among the chromosome in the groups.

$$
\begin{gathered}
d_{S}=\left\|S_{m i}-S_{n i}\right\|=\left[\sum_{j=1}^{n}\left(S_{m i j}-S_{n i j}\right)^{2}\right]^{\frac{1}{2}} \\
d_{T}=\left\|T_{m i}-T_{n i}\right\|=\left[\sum_{j=1}^{n}\left(T_{m i j}-J_{n i j}\right)^{2}\right]^{\frac{1}{2}}
\end{gathered}
$$

Where $S_{m i}$ and $S_{n i}$ are two chromosomes in the shelf stability; $n$ is the number of chromosomal genes; $T_{m i}$ and $T_{n i}$ are two chromosomes of cargo storage time efficiency.
From formula (5) and (6) can be seen that the greater two values of $d_{S}$ and $d_{T}$, the worse similarity between the two points. The smaller two values, the better similarity [8].

\section{The Fitness Function}

The two objective functions of aforementioned shelf stability and access time efficiency of cargo, are the questions of obtaining the minimum value. Establish the mapping relationship of following fitness function and the objective function.

$$
\begin{aligned}
& \begin{cases}f_{S}=S_{\max }-S & \text { if } S \leq S_{\max } \\
0 & \text { or }\end{cases} \\
& \begin{cases}f_{T}=T_{\max }-T & \text { if } \mathrm{T} \leq T_{\max } \\
0 & \text { or }\end{cases}
\end{aligned}
$$

$S_{\max }$ and $T_{\max }$ are the maximum values of all current generation functions in Formula (7) and (8).

\section{Selecting Crossover and Mutation}

(1) Selecting operating. When making selecting operating, the same number of two sub-populations is breed with the way of selecting in proportion to the fitness value, according to the two objective functions, and then merged to constitute the next generation population. When combining to form new population, choice will be labeled, and respectively showed the shelf stability or the principle of accessing efficient. Therefore, in order to ensure the unity of the two optimization goals, when individuals are chosen to the next generation population, the label " $\mathrm{S}$ " or " $\mathrm{T}$ " is especially labeled to show the difference. Selecting with elitist selection strategy, that is, after generating new population, if the fitness value of the best individual is less than the currently best individual's fitness value, then the individuals of current group fitness values greater than the next generation will be selected randomly to alternate to the next generation, which ensures that each generation of evolution achieves higher efficiency [13].

(2) cross-operating. The label on the individual is corresponding to the individual's gender, that is, cross operation develops only between individuals of two different sexes. This will ensure the adequate combination of good genes selected basing on two different objective functions, a compromise solution between the two goals. After implementation of the cross-pairing, cross-operating is completed according to the crossover probability, adopting cross partial match when crossover operator. Such as for two genes randomly select one of two mating split point, and composed of two division points $\left(X_{1}, Y_{1}\right),\left(X_{2}, Y_{2}\right)$, then make crossover operation on the matrix portion of the middle of the two points[13]. Fig.3 shows the operation process, selecting a matrix operator to make crossover operation. 


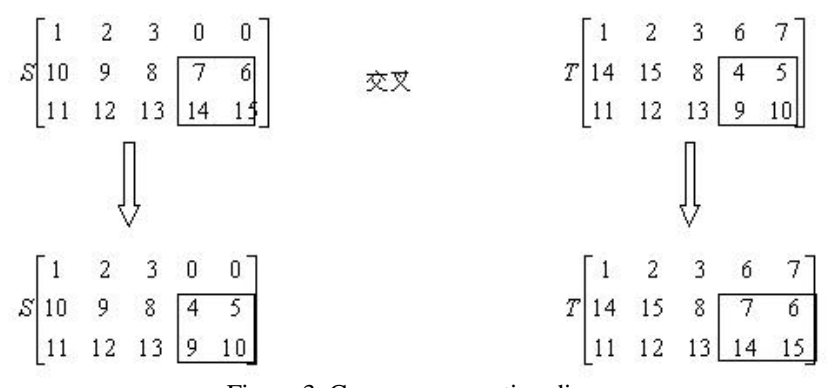

Figure 3. Crossover operation diagram

Mapping is done, to avoid the duplication of the cargo space, in order to legitimize the results after the crossover operation. As shown in Fig. 4:

$$
S\left[\begin{array}{ccccc}
1 & 2 & 3 & 0 & 0 \\
10 & 9 & 8 & 4 & 5 \\
11 & 12 & 13 & 14 & 10
\end{array}\right] \quad T\left[\begin{array}{ccccc}
1 & 2 & 3 & 4 & 5 \\
10 & 9 & 8 & 7 & 6 \\
11 & 12 & 13 & 14 & 15
\end{array}\right]
$$

Figure 4. Chromosomal after mapping

(3) The mutation operation. Mutation operation is variation implemented to select a part of the individual in accordance with a certain probability, typically in two ways: (1) Randomly select two cargo spaces, and change the items on the two cargo spaces, which is applied to a small range of variation, and selecting probability is generally between 0.01 to 0.05 , and the operation shown in Fig.5. (2) Randomly select a rectangular area, inverting the goods within the region. The way is applied to a wide range of variations, the probability of selection is generally 0.2 to 0.3 . The operation is shown in Fig.6.

$$
\begin{gathered}
T\left[\begin{array}{ccccc}
1 & 2 & 3 & 4 & 5 \\
10 & 9 & 8 & 7 & 6 \\
11 & 12 & 13 & 14 & 15
\end{array}\right] \\
T\left[\begin{array}{ccccc}
1 & 2 & 3 & 4 & 5 \\
10 & 9 & 6 & 7 & 8 \\
11 & 12 & 13 & 14 & 15
\end{array}\right]
\end{gathered}
$$

Figure 5. Small range of variation operator

$$
\begin{gathered}
T\left[\begin{array}{ccccc}
1 & 2 & 3 & 4 & 5 \\
10 & 9 & 8 & 7 & 6 \\
11 & 0 & 13 & 14 & 0
\end{array}\right] \\
T\left[\begin{array}{ccccc}
1 & 2 & 3 & 4 & 5 \\
10 & 9 & 14 & 13 & 6 \\
11 & 0 & 7 & 8 & 0
\end{array}\right]
\end{gathered}
$$

Figure 6. Large-scale variation operation

Emplacement rotation of the shelves and increasing the efficiency issues of passing in and out the goods are given in the variation operator. If certain location in the matrix is idle 0 , we can make use of variation running, and transposed to the top position of the same column shelf. As shown in Fig.7.

$$
T\left[\begin{array}{ccccc}
1 & 2 & 3 & 4 & 5 \\
10 & 9 & 14 & 13 & 6 \\
11 & 0 & 7 & 8 & 0
\end{array}\right] \Longrightarrow T\left[\begin{array}{ccccc}
1 & 0 & 3 & 4 & 0 \\
10 & 9 & 14 & 13 & 6 \\
11 & 2 & 7 & 8 & 5
\end{array}\right]
$$

Figure 7. Chromosome of cargo space repaired

\section{E. Simulation Analysis}

During the simulation analysis, warehouse is 5-layer and 20 -line, and the total number of cargos is 100 cargo spaces. The length of cargo space is $120 \mathrm{~cm}$, and the height of cargo space is $80 \mathrm{~cm}$. The level running speed of the shelf is $V_{x}=0.6 \mathrm{~m} / \mathrm{s}$, and the vertical running speed is $V_{y}=0.6 \mathrm{~m} / \mathrm{s}$. The average of each carton is limited to $100 \mathrm{~kg}$. When doing simulation experiments, the size of sample population is 100 , mutation probability 0.2 , crossover probability 0.8 , the maximum genetic algebra 50 .

Based on the optimization idea mentioned before, goods distribution is considered. The shelf area is divided into five types, respectively for the p1-p5. The way of goods storing has a great relationship with the efficiency of the accessing goods. The closer to the shipping, the more superior the cargo space is. Therefore, based on the foregoing analysis, cargo space arranged with fan-shaped. Cargo space followed with the classification principles, dividing into five categories of goods, depositing in five regions. Related parameters are shown in Table 1.

TABLE 1. EXPERIMENTAL PARAMETERS

\begin{tabular}{|l|l|l|l|l|l|}
\hline Item Number & A & B & C & D & E \\
\hline Share cargo median (a) & 4 & 8 & 12 & 19 & 17 \\
\hline Operation probability (\%) & 29 & 18 & 22 & 10 & 32 \\
\hline Quality (kg) & 100 & 80 & 60 & 50 & 30 \\
\hline Storage partitions & P1 & P2 & P3 & P4 & P5 \\
\hline
\end{tabular}

If using the way of traditional sequential storage, then $S$ is $3210 \mathrm{~kg}$, and $T$ is $621 \mathrm{~s}$. After optimization, $S$ is $2795 \mathrm{~kg}$, and $T$ is 571s. The analysis from the results was done, optimizing the storage order, significantly improving the stability of the shelf, and improving the efficiency of the accessing goods. Optimized cargo space distribution is shown in Fig.8.

\begin{tabular}{|l|l|l|l|l|l|l|l|l|lll|l|l|l|l|l|l|l|l|l|}
\hline P5 & P5 & P5 & P5 & P5 & P5 & P5 & P5 & P5 & P5 & P5 & P5 & P5 & P5 & P5 & P5 & P5 & P5 & P5 & P5 \\
\hline P5 & P5 & P5 & P5 & P4 & P4 & P4 & P4 & P4 & P4 & P4 & P4 & P4 & P4 & P4 & P4 & P4 & P4 & P4 & P4 \\
\hline P5 & P5 & P5 & P5 & P4 & P4 & P4 & P4 & P3 & P3 & P3 & P3 & P3 & P3 & P3 & P3 & P3 & P3 & P3 & P3 \\
\hline P5 & P5 & P5 & P5 & P4 & P4 & P4 & P4 & P3 & P3 & P3 & P3 & P2 & P2 & P2 & P2 & P2 & P2 & P2 & P2 \\
\hline P5 & P5 & P5 & P5 & P4 & P4 & P4 & P4 & P3 & P3 & P3 & P3 & P2 & P2 & P2 & P2 & P1 & P1 & P1 & P1 \\
\hline
\end{tabular}

Figure 8 space maps of cargo

\section{SUMMARY}

The principles and the general steps of slotting optimization were stated in detail, and using genetic algorithms to optimize the process were discussed. The 
general process of the optimization algorithm was designed. The simulation calculation of the stereo warehouse model was done, simulating optimally with the distribution of cargo space and based on the stability of the shelf, significantly improving the storage effect of the cargo.

\section{ACKNOWLEDGMENT}

This paper was sponsored by a key scientific research project of Shanghai (Project No. 11DZ2211104) and National Engineering Research Center of Protected Agriculture (Project No. 2011BAD43B01 and Project No. 2011BAD43B02).

\section{REFERENCES:}

[1] L.Y. Zheng, S.Y. Wang. Slotting Optimization Management of Automated Warehouse. Market Modernization, vol. 10, pp. 96, 2007.

[2] Y.L. Jia, L.X. Miu. Study on Optimization Problem of Cargo Space Real-time Allocation in the Automated Warehouse. Journal of Beijing Jiaotao University, vol. 4, pp.18-24, 2007.

[3] M. J. Li. Study on Optimization Methods of Automated Warehousing System. Dalian University of Technology, 2008.

[4] Lee H F, Schaefer S K. Retrieval Sequencing for Unit Load Automated Storage and Retrieval Systems With Multiple Openings. International Journal of Production Research, Vol. 34, pp. $2943-$ 2962, 1996
[5] C.J. Chen. Study on Intelligent Scheduling of Automated Warehouse. Mechanical Institute of Science and Technology, 2006.

[6] E Zitzler, L Thiele. Multi-objective Evolutionary Algorithm a Comparative Case Study and the Strength Pareto Approach. IEEE Trans on Evolutionary Computation, Vol. 3, pp. 257-271, 1999.

[7] Fonseca C M, Fleming P J. An Overview of Evolutionary Algorithms in Multi-objective Optimization. Evolutionary Computation, Vol.3, pp. 165-180, 1995.

[8] Y. Pan. Research on Automated Stereoscopic Warehouse Based on Modern Logistics. Suzhou University, 2008.

[9] J.G. Lu, Q. Li. Genetic Algorithm and Engineering Applications. Press of China Mining University, pp. 23-35,1997.

[10] H. Zheng. Study on Routing Optimization Problem of Automated Warehouse. Jilin University, 2006.

[11] Lina Sha. Management Methods of Slotting Optimization for Automated Warehouse. Dongbei University, 2005.

[12] Y.T. Chen, F. He. Slotting Optimization Allocation of the Automated Warehouse Based on Genetic Algorithm. Logistics Technology, vol. 1, pp. 38-40, 2008.

[13] D. Chen,X.Y. Liu. Applied Research on Genetic Algorithm in the Distribution of Cargo Space. Computer Knowledge and Technology, vol. 8, pp. 505-506, 2007.

[14] J. Zhang, D.L. Li, P. Li. Comparative Study of the Encoding Mechanism with Genetic Algorithm. Press of China Mining University,vol. 6, pp. 637-640, 2002. 